\title{
Diabetes, eating disorders and body image in young adults: an exploratory study about "diabulimia"
}

Maria Ana Falcão,

Rita Francisco, 1,2四

Phone +351217214199

Email ritafrancisco@fch.lisboa.ucp.pt

1 Faculdade de Psicologia, Universidade de Lisboa, Lisbon, Portugal

2 Faculdade de Ciências Humanas, Universidade Católica Portuguesa, Palma de Cima, 1649-023 Lisbon, Portugal

\section{Abstract}

\section{Purpose}

The purpose of this study was to compare disordered eating (DE) and body image dissatisfaction (BID) among young adults with type 1 diabetes and their peers without diabetes, to investigate the consequences of diabetes for food, body image and weight in individuals with diabetes and to identify the behavior of insulin omission as a weight loss strategy.

AQ1

$\mathrm{AQ} 2$

\section{Methods}

Fifty-five young adults with diabetes and 73 without diabetes (ages 18-30) completed self-report questionnaires to evaluate their behaviors, attitudes and feelings related to eating disorders and their perceptions about body image. The participants with diabetes were asked to answer a questionnaire with open and closed questions developed specifically for this study.

\section{Results}


No significant differences between participants with and without diabetes in relation to BID and DE were found. The results demonstrated several changes resulting from diabetes in terms of food, body image and weight that interfere with the day-to-day life of individuals with diabetes; $7.3 \%$ of these participants reported insulin omission as a weight loss strategy.

\section{Conclusions}

This study emphasizes the importance of research on DE in the population with diabetes and their prevention, screening and treatment. In particular, it is essential to give more attention to insulin omission as a compensatory behavior that is inappropriate and harmful to health.

\section{Keywords}

Diabetes

Disordered eating

Body image dissatisfaction

Omission of insulin

Diabulimia

Young adults

\section{Introduction}

Diabetes is a chronic disease (characterized by increased levels of glucose in the blood that occurs when the body does not produce enough insulin or cannot use it effectively $[1,2,3])$ that appears as a potential risk factor for the development of eating disorders (ED), due to its requirements with regard to food and weight control $[4,5,6]$. Patients with diabetes have a different relationship with their body and food when compared with other people, since the disease affects various daily activities in their life, including mealtimes [5]. Nutritionists' incentives for these patients to follow an eating plan can be a risk factor for ED. On the one hand, patients do not eat according to their sensations of hunger and satiety and, on the other hand, there is a major focus on food and calories that may imply restricting certain types of food $[4,7]$. 
"Diabulimia" is a term used to describe an ED in which the weight loss strategy involves intentional omission or limitation of insulin by patients with type 1 diabetes (T1DM) [5, 8, 9, 10,11], a type of diabetes for which it is necessary to administer daily insulin injections to control glucose levels in the blood [1, 2, 3, 12]. In several studies, this was the second most reported strategy among people with diabetes to lose weight, the first being dieting [13, 14, 15]. Patients with diabulimia present irregular eating patterns: on one hand, they avoid sweets and fats, restrict the food they eat and "skip" meals, to lose weight, and, on the other, they frequently ingest a large amount of food and feel guilty, then limit or omit insulin10. These patients quickly develop diabetic ketoacidosis, one of the most serious acute complications of diabetes which occurs when a person has elevated levels of blood glucose, lack of insulin in the body and an increase of hormones that counteract the action of insulin $[1,16,17,18]$. During this period, the cells cannot convert glucose from food into energy, so the body begins to burn fat for energy $[1,19]$ and the patient continues to lose weight $[8$, $11]$.

There is some controversy in the literature concerning the prevalence of ED in individuals with diabetes compared to their healthy peers, since some studies show an increased prevalence in people with diabetes [14] and others have found no significant differences [13]. Some studies show that major types of disordered eating in patients with T1DM are insulin omission as a weight loss strategy and binge eating $[14,15,20,21]$. As in the general population, women with diabetes show greater body image dissatisfaction (BID) than men [13, 22] and are at increased risk of developing ED [23], with insulin omission being used more frequently by women than men $[13,22]$.

Adolescents with diabetes are at greater risk of developing ED [23], especially adolescents with a lower desired weight [24]. Young people, and particularly young women, with T1DM are more concerned with their weight and body image as they consider it difficult to lose weight since the diagnosis of diabetes [25]. Some studies have found that, on average, patients with diabetes have a greater body mass index (BMI) than other people $[2,14,26]$. For example, almost $90 \%$ of individuals with diabetes in Portugal are overweight $(49.2 \%)$ or obese (39.6\%) [2]. Moreover, patients with greater BMI tend to be more dissatisfied with their body image, which can lead to inadequate strategies for weight control and increase the risk of developing ED [4, 22].

Insulin omission is initially perceived as a positive experience because patients 
lose weight without experiencing any visible side effects and, moreover, this behavior is often positively reinforced by compliments from family and friends [25]. These patients experience a feeling of mastery over their own bodies because they have discovered how to control their weight without others perceiving it [25], which makes them feel they have control over that behavior [7]. However, individuals get locked into this behavior because when they take their insulin correctly, they gain back all the weight they had previously lost, leading to an escalating situation in which they are not able to control their weight loss behaviors, and so these come to play an increasingly greater role in the patient's life [25]. Thus, these patients are unable to change their behavior even if they are aware of its consequences [25] because they ignore the longterm effects [8]. If health professionals are unable to proactively identify ED in patients with diabetes, this behavior may last for several years before patients seek help and may have serious consequences for the control of diabetes and mental health [25], which compromises both their physical and biopsychosocial well-being [27].

ED among individuals with T1DM increase the health risks associated with poor metabolic control of diabetes, which contributes to an increased risk of microvascular complications in these patients, such as nephropathy $[15,20,21$, 26], neuropathy [26], foot problems [20] and diabetic retinopathy [15, 21, 26]. Insulin omission is associated with a mortality risk which is three times higher in patients with diabetes, and the average age of death in these cases is lower [20]. Various studies also refer to other consequences of this behavior, such as late puberty, irregular or non-existent menstruation [10], problems with social physical anxiety [24], depressive episodes and suicidal thoughts [25, 28]. ED among individuals with diabetes also seem to be associated with negative and avoidant coping strategies [27].

Currently, there is little research on diabulimia and it is important, in particular, to gain a deeper understanding of what leads patients with diabetes to develop this weight loss strategy and what drives them to give it up $[6,20]$. Understanding the psychological mechanisms underlying diabulimia, on one hand, will enable an appropriate and effective psychological intervention to be outlined, and, on the other hand, will help to prevent the possible consequences of diabulimia in patients. Since most studies provide important information about this unhealthy behavior in women (especially adolescents) with T1DM, it is also important to study it in men and specifically in young adults. 
This research is a mixed methods study with two major objectives: (a) to characterize and compare young adults with diabetes and their peers without diabetes on BMI, BID and disordered eating (DE), and to evaluate possible predictor variables (sex, BMI, BID) of DE in both groups; (b) to explore the perceptions of young adults with T1DM about its consequences for food, body image and weight, and to identify the use of insulin omission behavior as a weight control strategy. Taking into account the results of previous studies, for the quantitative phase of this study (objective a), higher BMI, BID and disordered eating are expected in participants with diabetes than in the control group.

\section{Methods}

\section{Participants}

The study involved 128 young adults of both sexes, aged between 18 and 30 years: 55 participants with T1DM (18 men and 37 women; $M=24.78$ years, $\mathrm{SD}=4.18$ ), and 73 participants without diabetes (11 men and 62 women; $M=22.67$ years, $\mathrm{SD}=3.11)$. Eight participants without diabetes were reported to have chronic diseases like asthma $(n=6)$, lupus $(n=1)$ and Willebrand disease $(n=1)$. Among the participants with diabetes, the average age of diabetes onset was 13.85 years $(\mathrm{SD}=7.21)$ and the average duration of the disease was 10.93 years $(\mathrm{SD}=6.56)$. Some of them were also reported to have other chronic diseases, such as asthma $(n=2)$, epilepsy $(n=2)$ and renal insufficiency $(n=1)$.

AQ5

\section{Procedure}

Participants were recruited on the Internet using snowball sampling [29]. The inclusion criteria were: to be between 18- and 30-year-old; to be Portuguese or fluent in Portuguese (to answer the questionnaire); and to have T1DM (to integrate the group of participants with diabetes and to respond to the specific questionnaire). Exclusion criteria were, for both groups, to present other chronic diseases that could affect the diet and/or body image (e.g., celiac disease, T2DM). The link to participate was published on social networks and particularly in groups and associations of people with diabetes for these specific participants. The data were collected online through the Qualtrics platform after informed consent was obtained to ensure the anonymity of participants. 


\section{Measures}

\section{Demographic and Personal Data Questionnaire}

Participants completed a short questionnaire in which they reported personal data such as sex, age, nationality, educational qualifications, profession and weight, height and health problems (e.g., diabetes, other chronic diseases).

\section{Eating Disorder Examination-Questionnaire (EDE-Q) [30]}

This 28-item self-report questionnaire was used to measure the frequency of behaviors and attitudes related to ED in the last 28 days, to assess participants along a continuum of disordered eating. The EDE-Q scores provide an overall score that results from the average of the four subscales: Restraint, Eating Concern, Shape Concern and Weight Concern [30]. Higher scores indicate higher disordered eating. The Portuguese (male and female) version of the 5th edition of EDE-Q was used [31], which presents an excellent internal consistency of the four subscales $(\alpha=.84$ to $\alpha=.93)$ and the Global Score $(\alpha=.97)$. In the current study, the EDE-Q showed approximated internal consistency values (Global Score $\alpha=.95$, Shape Concern $\alpha=.92$, Weight Concern $\alpha=.80$, Restraint $\alpha=.84$ and Eating Concern $\alpha=.82$ ).

\section{Contour Drawing Rating Scale (CDRS) [32]}

It comprises nine male and nine female silhouettes numbered from one to nine, from severely underweight to extremely obese, in which body and face characteristics are defined. Each participant is asked to select the figure that most closely approximates his/her current body (current size) and the figure that he/she would most like to resemble (ideal size). The current ideal discrepancy indicates the level of BID, which can range from 8 (extreme desire to lose weight) to -8 (extreme desire to gain weight). No discrepancy between the current and ideal body image suggests a satisfaction with the body image. The Portuguese version was used, which presents good convergent validity: correlations of the silhouette representing current body size with weight $r=.65$ and with BMI $r=.72$ [33].

\section{Questionnaire on personal experience about food and body image}

The group of participants with T1DM answered a questionnaire developed specifically for this research. It sought to explore the perception that individuals with diabetes (and specifically insulin users) have on eating, weight and body image. It also aimed to evaluate deliberate insulin omission (e.g., reasons, 
duration, reaction of others, awareness of the consequences and support received). This is a 20-item self-report questionnaire with open questions (e.g., "What strategies do you use in your daily life to achieve the body image that you consider to be ideal for you?"; "What consequences do you think the omission of insulin may have in the short and long term for your health?") and with other questions answered in a 3- or 4-point Likert scale (e.g., "Do you consider that you take more care of your food than a person who does not have diabetes?"; "Since you have diabetes, do you find it more difficult to lose weight?").

\section{Data analyses}

Differences between individuals with and without diabetes were analyzed using independent samples $t$ tests. A Chi-square test was used to evaluate the differences in the clinically disordered eating indicator. Stepwise multivariate linear regression was used to identify factors associated with disordered eating in both study groups. The predictors included in the multivariate regression model were selected because they are three of the most common risk factors associated with the development of ED in the general population [38]. All quantitative analyses were performed with SPSS.

The open questions answered by participants with diabetes were subjected to an inductive-deductive content analysis procedure, using the software QSR NVivo 10. First, the answers were analyzed deductively for meaningful units, which were defined as sets of phrases about the same topic. Then, meaningful units that shared common features were identified and organized into distinct categories and combined to form more abstract categories, based on new labels or pre-existing concepts in the literature. Raw data responses were individually identified and grouped into themes and subthemes developed by the first author and checked for reliability and validity by the second author until a consensus was reached.

\section{Results}

Descriptive statistics and differences between young adults with and without diabetes

Table 1 shows the descriptive statistics of all study variables and the results of mean comparisons between the group of participants with diabetes and the control group. Participants with diabetes were significantly older and presented 
a significantly higher BMI than their healthy peers. No significant differences were found related to BID, the EDE-Q Global Score or its subscales.

\section{Table 1}

Descriptive statistics and results of mean comparisons between groups $(N=128)$

\begin{tabular}{|c|c|c|c|c|c|c|c|}
\hline \multirow[t]{2}{*}{ Variables } & \multirow[t]{2}{*}{ Range } & \multicolumn{2}{|c|}{$\begin{array}{l}\text { Participants } \\
\text { with diabetes } \\
(n=55)\end{array}$} & \multicolumn{2}{|c|}{$\begin{array}{l}\text { Participants } \\
\text { without diabetes } \\
(n=73)\end{array}$} & \multirow[t]{2}{*}{$t$} & \multirow[t]{2}{*}{$p$} \\
\hline & & $M$ & SD & $M$ & SD & & \\
\hline Age & & 24.78 & 4.18 & 22.67 & 3.11 & 3.277 & .001 \\
\hline BMI & & 24.13 & 3.90 & 22.03 & 3.48 & 3.199 & .002 \\
\hline $\begin{array}{l}\text { Shape } \\
\text { concern }\end{array}$ & $0-6$ & 1.73 & 1.82 & 2.02 & 1.54 & -.979 & .330 \\
\hline $\begin{array}{l}\text { Weight } \\
\text { concern }\end{array}$ & $0-6$ & 1.61 & 1.69 & 1.89 & 1.72 & -.909 & .365 \\
\hline Restraint & $0-6$ & 1.36 & 1.48 & 1.13 & 1.28 & .945 & .346 \\
\hline $\begin{array}{l}\text { Eating } \\
\text { concern }\end{array}$ & $0-6$ & 0.62 & 1.06 & 0.66 & 0.98 & -.212 & .832 \\
\hline $\begin{array}{l}\text { EDE-Q } \\
\text { Global Score }\end{array}$ & $0-6$ & 1.37 & 1.37 & 1.48 & 1.23 & -.489 & .626 \\
\hline BID & $\begin{array}{l}-8 \text { to } \\
8\end{array}$ & -1.11 & 1.57 & -1.15 & 1.54 & .149 & .882 \\
\hline
\end{tabular}

AQ6

\section{Clinical indication of DE}

According to the EDE-Q cutoff [34], 33 participants (25.8\% of the sample) showed a clinical level of DE, specifically $29.1 \%$ of participants with diabetes and $23.3 \%$ of participants without diabetes. However, this difference was not statistically significant $\left(\chi^{2}(1)=.552, p=.457\right)$, indicating that there was no association between the group of participants and the clinical indication of DE.

\section{Initial age and duration of diabetes}

No significant correlations were found between the initial age of diabetes with BMI $(r=-.013, p=.925)$, BID $(r=-.060, p=.665)$, EDE-Q Global Score and 
its subscales $(r=.007$ to $r=.148, p>.05$ ), or between duration of diabetes with BMI $(r=.163, p=.236)$, BID $(r=.061, p=.660)$, EDE-Q Global Score or its subscales $(r=-.025$ to $r=.165, p>.05)$.

\section{Predictors of DE}

The results summarized in Table 2 show that in the group with diabetes, only BID proved to be a significant predictor of DE. This model explains $51 \%$ of the variance of $\mathrm{DE}$ in participants with diabetes, to which negative values of BID contribute. In the control group, BMI proved to be a significant predictor of DE. The model explained $39.4 \%$ of the variance of DE among participants without diabetes, to which high BMI contributes.

\section{Table 2}

Summary of linear regression analyses of variables predicting participants' DE (EDEQ Global Score) by group

\begin{tabular}{|c|c|c|c|c|c|c|}
\hline \multirow[t]{2}{*}{ Variables } & \multicolumn{3}{|c|}{$\begin{array}{l}\text { Participants with diabetes } \\
(n=55)\end{array}$} & \multicolumn{3}{|c|}{$\begin{array}{l}\text { Participants without diabetes } \\
(n=73)\end{array}$} \\
\hline & B & SE B & $\beta$ & B & SE B & $\beta$ \\
\hline $\operatorname{Sex}^{\mathrm{a}}$ & .075 & .326 & .026 & .520 & .338 & .153 \\
\hline BMI & -.027 & .047 & -.076 & .174 & .049 & $.487 * *$ \\
\hline BID & -.658 & .115 & $-.751 * * *$ & -.125 & .110 & -.157 \\
\hline$R^{2}$ & \multicolumn{3}{|l|}{.510} & \multicolumn{3}{|l|}{.394} \\
\hline $\mathrm{F}$ & \multicolumn{3}{|c|}{$17.343 * * *$} & \multicolumn{3}{|c|}{$14.744 * * *$} \\
\hline \multicolumn{7}{|c|}{$B M I$ body mass index, $B I D$ body image dissatisfaction } \\
\hline \multicolumn{7}{|c|}{ a Sex of participants $($ men $=0$, women $=1)$} \\
\hline \multicolumn{7}{|c|}{$* * p<.01 ; * * * p<.001$} \\
\hline
\end{tabular}

\section{Personal experience of individuals with diabetes about food and body image}

Thematic analysis of responses to the open questions resulted in 43 interrelated categories organized in a hierarchical system with seven main categories.

Regarding the Changes resulting from diabetes, most participants considered that they take greater care with food $(47.2 \%$ of participants reported taking a 
little care and $37.7 \%$ said they were very careful) and some participants considered that now they take greater care with their body image, while patients with diabetes $(26.4 \%)$. felt

(...) I ended up paying more attention to what I eat so you can keep the blood glucose levels under control. (29-year-old man, student)

The care with body image is no different in a person with diabetes compared to a person without diabetes. (29-year-old man, unemployed)

Some participants reported having greater difficulty losing weight since they were diagnosed with diabetes $(35.8 \%)$, a situation that all of them considered a cause for concern.

Sometimes the food I eat is excessive, but it is necessary to get the values under control. (24-year-old woman, student)

In relation to Strategies that participants use in their daily lives to achieve the ideal body image, they referred to Physical exercise $(51.5 \%$ of the coded references), Food (42.-6\%), Insulin omission (1.5\%) and Other strategies $(4.4 \%)$ :

I don't eat carbohydrates or when I eat them I don't use insulin (24-year-old woman, office manager).

As for the participants' perceptions regarding the influence of insulin on body weight, most participants considered that insulin was not fattening $(67.9 \%$ of participants) and the remaining participants (32.1\%) felt a weight fluctuation before and after diagnosis.

The effect of insulin is to lower glucose; what makes you fat is eating every hour to have carbohydrate control. (25-year-old woman, kitchen assistant)

With respect to Consequences of insulin omission, participants reported as 
Short-Term Consequences (41.8\%) the poor metabolic control of diabetes, in particular, related hyperglycemia and diabetic ketoacidosis, and there were also two references to losing weight. As Long-Term Consequences (58.2\%), participants reported most frequently retinopathy, neuropathy, nephropathy, cardiovascular disease and limb amputations.

I know that omitting insulin doses causes weight loss because of the process that develops in the body. (30-year-old woman, facilitator)

Eleven participants with diabetes (20.8\%) reported having stopped taking insulin intentionally, four of whom ( $7.3 \%$ of participants with diabetes) said they did so specifically to lose weight. These participants $(n=4)$ were all women, aged between 18 and 30 years $(M=22.75, \mathrm{SD}=5.50)$, with normal weight, dissatisfied with their body image and wishing to lose weight, and showed significant clinical indication of DE. The four participants considered that they were more careful with their food and body image as patients with diabetes, and also reported having greater difficulty losing weight since they were diagnosed with diabetes.

When I start to do the right control, measure, use the correct dosage, I get too fat (...) I start to eat more and not take insulin, I have long periods without [insulin] to get into ketosis [ketoacidosis]. (24-year-old woman, office manager)

Initially it increased my appetite and now I am more careful with the food I eat but I cannot lose weight. (30-year-old woman, banker)

The participants stated that when they stopped taking insulin, they were aware of the consequences of this behavior. As for the Reasons to ignore the consequences, the responses indicated the desire to lose weight and feelings of resentment.

I don't think about it... I know all [the consequences] but I think that what matters to me is to be thin (...) I don't feel good being fat. (24-year-old woman, office manager) 
Because it was a simple way to lose weight while eating what you wanted or without much exercise. (19-year-old woman, student)

Regarding the duration of insulin omission, two participants reported a longer and continued period of insulin omission, while another participant said that insulin omission was occasional.

The most I can go is a week with nothing [insulin]... Now I've been months without using the fast [insulin]... I lost $10 \mathrm{~kg}$. (24year-old woman, office manager)

Two years, but I'm taking some insulin to feel better. (19-yearold woman, student)

As for support felt by participants for dealing with insulin omission (Social Network), the family (e.g., parents, siblings, spouse or boyfriend/girlfriend) was often mentioned, as well as doctors and friends.

... my mother. [She was] always by my side even though it was a terrible period due to the vigilance because she was by controlling my diabetes and always being suspicious, but she was the only person from whom I felt support and care. (19year-old woman, student)

\section{Discussion and conclusions}

This study aimed to characterize and compare young adults with and without diabetes in terms of BMI, BID and DE, and to evaluate possible predictor variables (sex, BMI, BID) of DE in both groups. It also aimed to explore the consequences of diabetes for food, body image and weight in patients with diabetes, and identify the behavior of insulin omission as a weight loss strategy.

The results confirmed that individuals with diabetes have a higher BMI compared to their peers without diabetes $[2,14,26]$, which may be due to the influence of intensive treatment of diabetes on patients' body weight [35]. However, the absence of significant differences between participants with and 
without diabetes in relation to BID did not confirm the negative effects of diabetes on body image described in the literature [4, 22]. A possible explanation for both of these results is the higher percentage of male participants in the group with diabetes, compared to the control group, since previous studies [33] have shown that males are more satisfied with their body image than females.

Considering sex, $\theta f \mathrm{BMI}$ and BID as possible predictors of DE, as suggested by other authors $[4,22,36,37,38]$, curiously only BID proved to be a significant predictor of DE in the group with diabetes and BMI in the control group. The fact that BMI is not a significant predictor in the group with diabetes may be due, as referred above, to the fact that this group has a higher percentage of male participants than the control group. However, it was interesting to note that the sex of the participants was not a predictor of DE (neither among the participants with diabetes nor in the control group), since we know that being a woman is a risk factor for the development of these types of problems [38]. In the same direction, the fact that the BID in the control group was not a predictor of $\mathrm{DE}$ is inconsistent with the majority of the literature [36, 37], where the search for the current ideal of beauty and the discrepancy between the real and ideal body image are associated with an increased risk of DE, especially for women. However, the absence of significant differences in clinical indication of DE among individuals with diabetes and their healthy peers suggests that our sample of participants with diabetes may have a nutritionally and/or medically appropriate treatment, as found by Ackard et al. [13].

The changes resulting from diabetes reported most frequently by participants with diabetes were related to food, since the majority of the participants reported paying greater attention to diet since diagnosis, which confirms that diabetes affects their daily life activities involving food [5]. They also reported more exercise and more attempts at weight control [28]. $\mathrm{AQ7}$

In this study, a considerable number of participants reported taking greater care with their body image and having difficulty losing weight since they were diagnosed with diabetes (26.4 and $35.8 \%$, respectively), which is consistent with previous studies [25, 35]. One of these studies [35] demonstrated that the use of insulin is commonly associated with weight gain, whereas in our sample about one-third of the participants reported that association, and some participants reported a weight fluctuation before and after diagnosis. Our study 
showed that the strategies most used by young people with diabetes to achieve their ideal body image are physical exercise and nutritional care, as noted in the general population [35]. Intentional insulin omission was also referred to as a weight loss strategy, as already reported in several previous studies $[6,13,14$, $15,20,21,22,25,26,28,39,40]$.

In our study, $7.3 \%$ of individuals with diabetes reported omitting insulin to lose weight, and similar results were found by other recent studies $[13,22]$. Other studies $[14,15,20,25,26]$ found a higher percentage of individuals with diabetes using this weight loss strategy, although these studies were carried out only with females or with a very small percentage of males. The participants with diabetes who reported insulin omission as a weight loss strategy are only women, which is consistent with previous studies [13, 22, 25]. Besides the body image dissatisfaction and the desire to lose weight, these participants have a significant clinical indication of DE, confirming the results of previous studies that the omission of insulin as a weight loss strategy is possibly associated with ED $[13,22]$. These participants take extra care with food and their body image, and also have greater difficulty losing weight since the diagnosis, a situation they consider a cause for concern, which can be considered risk factors for the development of this compensatory behavior.

In the present study, was found that most individuals with diabetes know the effects that insulin omission can cause, since they reported hyperglycemia and diabetic ketoacidosis as short-term consequences and microvascular complications as long-term consequences, which are the most frequently mentioned consequences in the literature [1, 2, 3, 41]. However, most participants who omit insulin to lose weight reported ignoring these consequences because of the importance that the weight loss has in their life. One reason for continuing this compensatory behavior is that once the individuals take insulin correctly again, they gain back all the weight they had previously lost, a fact that was also reported in previous research [25]. The family and the medical team often appear as a source of support in cases of insulin omission for weight control. However, it is important to pay special attention to cases in which the presence of this behavior is ignored. In these cases, the association between insulin omission and DE is not recognized, since only the metabolic control of the patient is given attention [6].

Our study extended previous research, combining quantitative and qualitative data to compare DE and BID among individuals with diabetes and their peers 
without diabetes, and to show the consequences of diabetes on the food, body image and weight of young adults with T1DM. To our knowledge, this is the first study that compares these variables among the Portuguese population with and without diabetes. Also, the fact that the group with diabetes includes men allows us to better assess this understudied subsample.

There are some limitations, however, that should be mentioned. Firstly, some specific limitations were related to the constitution of groups. On the one hand, the participants with diabetes were significantly older than the control group and the percentage of men in the group with diabetes was twice as high as that in the control group. This could have affected the results, since there is a consensus in the literature that ED are more common in women [38] and in younger people $[23,38]$. This unequal distribution with respect to the gender and the age of participants possibly can have increased the frequency of ED in the control group compared to the group of participants with diabetes. On the other hand, the participants of the control group could have some health problems (not identified as chronic diseases) that were not controlled in the study and it can influence their body image and their behaviors and attitudes about eating in the same way. Both of these aspects should be considered in future studies. Secondly, the self-report instruments increase the possibility of responses by social desirability or at random, especially if answered online; so the additional use of other sources of information (e.g., health professionals) could be useful to assess more precisely the cases of insulin omission as DE. Thirdly, considering the small number of participants who reported insulin omission as a weight loss strategy, it was not possible to explore as deeply as necessary this risk behavior, which characterizes diabulimia. Moreover, the instruments used to assess the risk of ED are not specific for patients with diabetes, so the results comparing participants with and without diabetes should be read with caution. Hence the construction, translation or validation of specific tools to assess insulin omission as DE in Portuguese population with diabetes would be important. It is important that future research should check the existence of generational differences regarding insulin omission as a weight loss strategy, particularly examining this ED in adolescents, since they are a risk group $[23,38]$.

The prevention of ED should be considered within the population with diabetes, because changes in diet and weight that patients feel from the diagnosis can be risk factors. Screening in this population, especially for females, is an effective way to promote early diagnosis and proper treatment of unhealthy eating and 
weight loss strategies and thus prevent future complications of diabetes. It is also crucial that health professionals are more aware of the first signs (e.g., recurrent episodes of diabetic ketoacidosis, elevated blood glucose values for no apparent reason and rapid weight loss). Psychological support should be a key part of the treatment to offer patients the necessary conditions for recovery and minimize the consequences for their mental health.

\section{Acknowledgements}

This study was partially supported by the Research Center for Psychological Science (UID/PSI/04527/2013) of the Ministry of Science and Technology (FCT, Portugal). The authors would like to express appreciation to all the participants.

Compliance with ethical standards

Conflict of interest On behalf of all authors, the corresponding author states that there is no conflict of interest.

Ethical approval All procedures performed in studies involving human participants were in accordance with the ethical standards of the institutional and/or national research committee and with the 1964 Helsinki Declaration and its later amendments or comparable ethical standards.

Informed consent Informed consent was obtained from all individual participants included in the study.

\section{References}

1. International Diabetes Federation (2013) IDF diabetes atlas, 6th edn. International Diabetes Federation, Brussels

2. Observatório Nacional da Diabetes (2014) Relatório anual do observatório nacional da diabetes [Annual report of the national diabetes observatory]. Sociedade Portuguesa de Diabetologia, Lisboa

3. World Health Organization (1999) Definition, diagnosis and classification of diabetes mellitus. World Health Organization, Geneva

4. Colton P, Rodin G, Bergenstal R, Parkin C (2009) Eating disorders and 
diabetes: introduction and overview. Diabetes Spectr 22(3):138-142. doi:10.2337/diaspect.22.3.138

5. Menina A, Akers EM (2014) Diabulimia: not so secret-or shamefulanymore. Diabetes Health 23(1):14-19

6. Powers MA, Richter S, Ackard D, Gerken S, Meier M, Criego A (2012) Characteristics of persons with an eating disorder and type 1 diabetes and psychological comparisons with persons with an eating disorder and no diabetes. Int J Eat Disord 45(2):252-256. doi:10.1002/eat.20928

7. Weaver K (2012) Eating disorders in people with type 1 diabetes. Nurs Stand 26(43):43-47

8. Darbar N, Mokha M (2008) Diabulimia: a body-image disorder in patients with type 1 diabetes mellitus. Athl Ther Today 13(4):31-33

9. Davidson J (2014) Diabulimia: how eating disorders can affect adolescents with diabetes. Nurs Stand 29(2):44-49

10. Shih GH (2009) Diabulimia. Diabetes Health 18(2):14-21

11. Shih GH (2011) Once a spokesperson for juvenile diabetes, Erin now suffers from diabulimia. Diabetes Health 20(4):10

12. Direção Geral de Saúde (2011) Diagnóstico e classificação da diabetes mellitus [Diagnosis and classification of diabetes mellitus]. Direção Geral de Saúde, Lisboa

13. Ackard DM, Vik N, Neumark-Sztainer D, Schmitz KH, Hannan P, Jacobs DR Jr (2008) Disordered eating and body dissatisfaction in adolescents with type 1 diabetes and a population-based comparison sample: comparative prevalence and clinical implications. Pediatr Diabetes 9(4 Pt 1):312-319. doi:10.1111/j.1399-5448.2008.00392.x

\section{Jones JM, Lawson ML, Daneman D, Olmsted MP, Rodin G (2000)} Eating disorders in adolescent females with and without type 1 diabetes: cross sectional study. BMJ 320(7249):1563-1566 
15. Rydall AC, Rodin GM, Olmsted MP, Devenyi RG, Daneman D (1997)

Disordered eating behavior and microvascular complications in young women with insulin-dependent diabetes mellitus. N Engl J Med 336(26):1849-1854. doi:10.1056/NEJM199706263362601

16. Umpierrez GE, Murphy MB, Kitabchi AE (2002) Diabetic ketoacidosis and hyperglycemic hyperosmolar syndrome. Diabetes Spectr 15(1):28-36. doi:10.2337/diaspect.15.1.28

17. Wolfsdorf J, Craig ME, Daneman D, Dunger D, Edge J, Lee W et al (2009) Diabetic ketoacidosis in children and adolescents with diabetes. Pediatr Diabetes 10(Suppl 12):118-133. doi:10.1111/j.13995448.2009.00569.x

18. Wolfsdorf JI, Allgrove J, Craig ME, Edge J, Glaser N, Jain V et al (2014) Diabetic ketoacidosis and hyperglycemic hyperosmolar state. Pediatr Diabetes 15(Suppl 20):154-179. doi:10.1111/pedi.12165

19. American Diabetes Association (2013) DKA (ketoacidosis) and ketones. IOP Publishing Diabetes. http://www.diabetes.org/living-withdiabetes/complications/ketoacidosis-dka.html?loc $=$ lwd-slabnav. Accessed 28 Novemb 2016

20. Goebel-Fabbri AE, Fikkan J, Franko DL, Pearson K, Anderson BJ, Weinger K (2008) Insulin restriction and associated morbidity and mortality in women with type 1 diabetes. Diabetes Care 31(3):415-419. doi: $10.2337 / \mathrm{dc} 07-2026$

21. Takii M, Uchigata Y, Tokunaga S, Amemiya N, Kinukawa N, Nozaki T et al (2008) The duration of severe insulin omission is the factor most closely associated with the microvascular complications of type 1 diabetic females with clinical eating disorders. Int J Eat Disord 41(3):259-264. doi:10.1002/eat.20498

22. Philippi ST, Cardoso MGL, Koritar P, Alvarenga M (2013) Risk behaviors for eating disorder in adolescents and adults with type 1 diabetes. Rev Bras Psiquiatr 35(2):150-156. doi:10.1590/1516-4446-2012-0780

23. Peveler R (2000) Eating disorders and insulin-dependent diabetes. Eur 
Eat Disord Rev 8(2):164-169. doi:10.1002/(SICI)1099-

0968(200003)8:2<164:AID-ERV345>3.0.CO;2-V

24. Gonçalves S, Barros V, Rui Gomes A (2016) Eating-disordered behaviour in adolescents with type 1 diabetes. Can J Diabetes 40(2):152157. doi:10.1016/j.jcjd.2015.09.011

25. Balfe M, Doyle F, Smith D, Sreenan S, Conroy R, Brugha R (2013) Dealing with the devil: weight loss concerns in young adult women with type 1 diabetes. J Clin Nurs 22(13-14):2030-2038. doi:10.1111/jocn.12231

26. Peveler RC, Bryden KS, Neil HA, Fairburn CG, Mayou RA, Dunger DB et al (2005) The relationship of disordered eating habits and attitudes to clinical outcomes in young adult females with type 1 diabetes. Diabetes Care 28(1):84-88

27. Grylli V, Wagner G, Hafferl-Gattermayer A, Schober E, Karwautz A (2005) Disturbed eating attitudes, coping styles, and subjective quality of life in adolescents with type 1 diabetes. J Psychosom Res 59(2):65-72. doi:10.1016/j.jpsychores.2005.02.010

28. Colton PA, Olmsted MP, Daneman D, Rodin GM (2013) Depression, disturbed eating behavior, and metabolic control in teenage girls with type 1 diabetes. Pediatr Diabetes 14(5):372-376. doi:10.1111/pedi.12016

29. Heckathorn D (1997) Respondent-driven sampling: a new approach to the study of hidden populations. Soc Probl 44:174-199. doi: $10.2307 / 3096941$

30. Fairburn CG, Beglin SJ (1994) Assessment of eating disorders: interview or self-report questionnaire? Int J Eat Disord 16(4):363-370

31. Machado PP, Martins C, Vaz AR, Conceição E, Bastos AP, Gonçalves S (2014) Eating disorder examination questionnaire: psychometric properties and norms for the portuguese population. Eur Eat Disord Rev 22(6):448453. doi:10.1002/erv.2318

32. Thompson MA, Gray JJ (1995) Development and validation of a new body-image assessment scale. J Pers Assess 64(2):258-269 
33. Francisco R, Alarcão M, Narciso I (2012) Body image (dis)satisfaction among Portuguese adolescents and adults: contribution to the validation process of the Contour Drawing Rating Scale. Revista Iberoamericana de Diagnóstico y Evaluación Psicológica 34(1):61-88

34. Fairburn CG, Cooper Z (1993) The eating disorder examination. In: Fairburn CG, Wilson GT (eds) Binge eating: nature, assessment and treatment, 12th edn. The Guilford Press, New York, pp 317-331

35. Diabetes Control and Complications Trial Research Group (2001) Influence of intensive diabetes treatment on body weight and composition of adults with type 1 diabetes in the diabetes control and complications trial. Diabetes Care 24(10):1711-1721

36. Striegel-Moore RH, Bulik CM (2007) Risk factors for eating disorders. Am Psychol 62(3):181-198

37. Striegel-Moore RH, Debra FL (2002) Body image issues among girls and women. In: Cash T, Pruzinsky T (eds) Body image: a handbook of theory, research and clinical practice. Guilford Press, New York, pp 183-191

38. Fairburn CG, Harrison PJ (2003) Eating disorders. Lancet 361(9355):407-416. doi:10.1016/S0140-6736(03)12378-1

39. Custal N, Arcelus J, Agüera Z, Bove FI, Wales J, Granero R et al (2014) Treatment outcome of patients with comorbid type 1 diabetes and eating disorders. BMC Psychiatry 14:26-37. doi:10.1186/1471-244X-14-140

40. Olmsted MP, Daneman D, Rydall AC, Lawson ML, Rodin G (2002) The effects of psychoeducation on disturbed eating attitudes and behavior in young women with type 1 diabetes mellitus. Int J Eat Disord 32(2):230-239

41. World Health Organization (1994) Prevention of diabetes mellitus: report of a WHO study group. World Health Organization, Geneva 